\title{
Arzneitees und Phytopharmaka für den Magen
}

\author{
Klagt ein Patient über Übelkeit, Erbrechen und unspezifische Magen- \\ beschwerden, können naturheilkundliche Therapien oft viel bewirken. \\ Prof. Beer empfiehlt - je nach Symptomatik - verschiedene Arzneitees \\ und Fertigarzneimittel.
}

den Arzneitees beispielsweise Fencheloder Kamillentee zu nennen, bei den Fertigarzneimitteln Ingwer, der in Form eines Extraktpräparates in der Apotheke erhältlich ist.

Treten Übelkeit und Erbrechen als Teil der Symptomatik einer funktionellen Dyspepsie auf, also vergesellschaftet mit anderen Magenbeschwerden wie Völlegefühl im Oberbauch, Appetitlosigkeit oder saurem Aufstoßen, dann sind neben den genannten Arzneitees pflanzliche Fertigarzneimittel die erste Empfehlung.

Als eines der immer noch relativ wenigen pflanzlichen Arzneimittel, die in die gastroenterologischen Leitlinien aufgenommen wurden, ist hier der Extrakt (STW 5) von Iberis amara und acht weiteren pflanzlichen Extrakten zu nennen, der in Studien eine hohe Evidenz bei dieser Indikation gezeigt hat. Das Flüssigarzneimittel kann auch bei Kindern ab drei Jahren empfohlen werden und wird dreimal täglich vor oder nach den Mahlzeiten oder bei Bedarf eingenommen.

Ebenfalls durch Studien bei Magenbeschwerden belegt ist die Anwendung der Kombination von Pfefferminz- und Kümmelöl, die in Kapselform vorliegt.

Stehen, bei Fehlen von säurebedingten Symptomen, postprandiale Beschwerden im Vordergrund, kommen auch Bitterstoffpräparate in Frage. Hier ist die Anwendung von Artischockenextrakt durch Studien belegt. Bei anderen Bitterstoffkombinationen, die beispielsweise Extrakte von Angelikawurzel und Benediktenkraut enthalten, stützt sich die Anwendung auf eine langjährige Tradition.

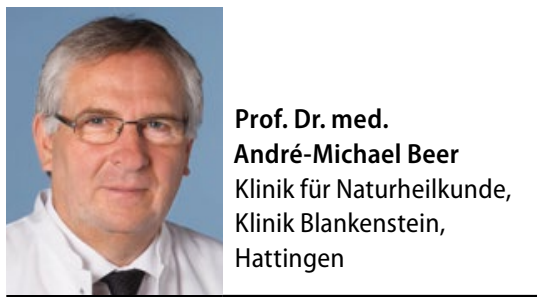

\section{Erbrechen in der Schwangerschaft}

Zu Ingwer gibt es klinische Studien, die auch seine Wirksamkeit bei Schwangerschaftserbrechen gezeigt haben. Es gibt zwar keine Hinweise darauf, dass Ingwer ungünstige Wirkungen auf den Embryo haben könnte, jedoch reichen hier wie bei fast allen anderen pflanzlichen Wirkstoffen die Daten nicht aus, um die Anwendung in der Schwangerschaft in die Zulassung aufzunehmen.

Die Phytotherapie hält demnach eine Vielzahl an Therapiemöglichkeiten bereit, die insbesondere dann zum Einsatz kommen können, wenn für Erbrechen, Übelkeit und Magenbeschwerden mit den üblichen diagnostischen Verfahren keine organischen Ursachen identifiziert werden können. Vorteilhaft ist auch, dass diese Mittel mit dem grünen Rezept verordnet werden können, also nicht das Arzneimittelbudget belasten, und eine hohe Akzeptanz beim Patienten aufweisen.

MEHR ZUM THEMA AUF SPRINGERMEDIZIN.DE

Wie Sie an typische Beratungsanlässe in Ihrer Praxis aus naturheilkundlicher Sicht herangehen, erfahren Sie in diesem Dossier von ausgewiesen Experten der Naturheilkunde.

$\rightarrow$ www.springermedizin.de/239812 